\title{
138. The Dip-prism Microscope, Specially Designed for Electrophysiological Use
}

\author{
By Yasuji Katsuki, Nobuo Suga, Masahiro Nomoto,** \\ and Toshio Nakatsubo**) \\ (Comm. by Y. Kuno, M.J.A., Nov. 13, 1961)
}

When the transmembrane potentials are recorded from cells situated deeply in a tissue, for example, from neurons in the central nervous system of higher animals, it is highly desired to know from what sort of cell, or particularly from what part of a cell the potentials are recorded. Based on the anatomical knowledge an electrode is simply forwarded into a tissue during delivery of the ortho- or anti-dromic stimulation to the neurons and the cell inserted by the electrode is indirectly identified by electrical signs. Since the discovery of a superfine microelectrode the present authors have not heard nevertheless the attempt of recording the electrical activity of cell in the tissue under visual control. After many trials, however, the present authors have finally succeeded in recording the electrical activity of some large neurons by using a specially designed "dip-prism microscope". In this preliminary report the principle of the microscope and some results obtained with it will be described.

The dip-prism microscope. In order to observe the cells located: deeply in the tissue, firstly the light has to be brought into there without serious damage of the tissue. For this purpose, the illuminator with a fine tip was devised. A glass rod of $8 \mathrm{~mm}$ in diameter is rapidly pulled after being softened by heat and the glass rod with a shape as shown in Fig. 1. A is made (Illuminator). The big end is flattened at right angles to the axis of the rod, while the thin end is polished at about 30 degrees to the axis as shown in the inset of Fig. 1.A (I). The image of the filament of a tungsten lamp as a light source is focused with a lens on the big flattened end after passing through a heat insulating filter. All of the light passing through the big end is led by reflexion to the thin polished end without escaping into the air from the side wall of the rod and goes out from this extreme end. The illuminator thus plays a role of a light condenser. When the tip of the illuminator is immersed in water, however, the light coming into the illuminator from the big conical end, leaks out through the side wall contacting with water because of the similar refractive index of water to that of the glass

*) Department of Physiology Tokyo Medical and Dental University, Tokyo.

**) Olympus Optical Company, Tokyo. 


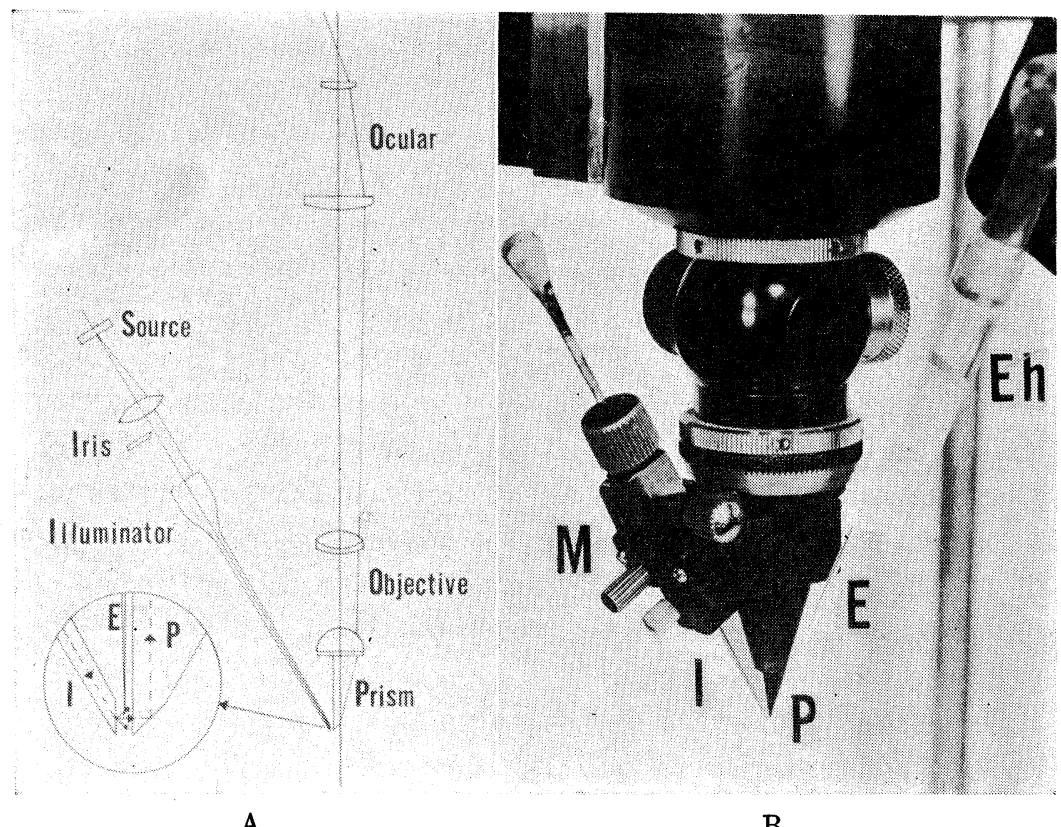

A

B

Fig. 1. A: Schematic representation of the dip-prism microscope.

E: Microelectrode, I: Illuminator, P: Prism.

B: Parts of the dip-prism microscope, Eh: Electrode-holder.

M: Micromanipulator.

and the light goes scarcely out through the thin tip. In order to prevent such leakage, the side wall of the illuminator is coated with aluminium in a vacuum chamber by evaporation except the thin polished end. Furthermore this coat is strengthened by being covered with a thin layer of polyethylene lacquer, in order to protect it against the erosive action of the salts in the Ringer's solution. The diameter of the tip is made from 50 to $200 \mu$ at will. By inserting this illuminator into the tissue, cells around its tip can be illuminated brightly.

Nextly a very fine prism is fixed on the objective $(\times 10)$ of an ordinary microscope. By this device the working distance of the microscope lengthens and the tip of the prism can reach the layer deeply as being wanted. The schematic view of the prism is shown in Fig. 1. A. Further the details of the prism is seen in the inset (P). As shown in the inset the tip of the prism is polished up at 45 degrees to the vertical axis and this polished surface is coated with aluminium to be a mirror. This mirror is also protected from the affection of salt solution or tissue fluid with a thin layer of polyethylene lacquer. The mirror is between $50 \times 200 \mu^{2}$ and $25 \times$ $100 \mu^{2}$ in its area and about $7.7 \mathrm{~mm}$ distant from the objective. When the prism is inserted into a tissue, the image of the illuminated cell 
is projected on the mirror and then reflected upwards. Thus we can see it through the ocular.

Actually, the illuminator is held at the side of the objective with a small micromanipulator (M) in Fig. 1. B which can move freely in three dimensions, and it is preliminarily set up in the air by handling the micromanipulator so as to get good illumination (Fig. 1. B). The tip of the illuminator is usually distant less than $0.3 \mathrm{~mm}$ from the prism as shown in the inset of Fig. 1. A. After such an adjustment, the prism and the illuminator are inserted into the tissue by the coarse and fine adjustments of the microscope. Whenever cells come between the tip of illuminator and the prism, we can observe them. The focus of the microscope is adjusted by pulling up or pushing down the draw tube. The illuminator is readjusted by the micromanipulator so as to make the image of the cell clearer. Thus the sharp image can be obtained.

In electrophysiological experiments it is further desired to insert a microelectrode into the cell under visual control. The other micromanipulator is fixed at the body tube of the microscope. A glass microelectrode is kept with a plastic, electrically insulated electrodeholder mounted on the micromanipulator (Fig. 1. B). After setting up all the equipments so as the tip of the microelectrode is observed on the mirror of the prism in the air, the electrode is drawn back quite a bit and then the prism is sent into the tissue together with the illuminator and the electrode. When the cell is visualized on the mirror, the electrode is pushed forward to it by handling the micromanipulator.

Experiments. It was finally tried to record impulse discharges from a nerve cell with this microscope. For the stimulation an electronic circuit reported by Ito (1) was adopted, that is, a single capillary microelectrode was used as a stimulating and also a recording one. At first the ganglion cell of a mollusc (Onchidium verruculatum) was used as a material, taking advantage of a large size of the cell body (about 100-150 $\mu$ in diameter). The biggest cell occupied almost all area of the mirror. The image of the cell was reflected by the wall and two or three or four images were observed by different positions of the tip of the illuminator. The eyes were kept on the best image among them and a capillary electrode filled with $3 \mathrm{M}-\mathrm{KCl}$ solution was inserted into the cell under visual control. The process that the microelectrode impaled the cell membrane was observed at hand and the resting potential of the cell was measured. When the electric current was flowed out from the electrode in such a way as to depolarize the cell membrane, spike potentials were recorded from the cell. No change in the resting and spike potentials were 
found for 30 minutes in spite of long maintained illumination.

Further trials to record the electrical activity from smaller cells were performed on the frog's spinal ganglion VIII or IX desheathed. As the diameters of those ganglion cells used ranged between 40 and $50 \mu$, the impalement of the cell membrane was easily done with a microelectrode (Fig. 2. A) and the resting potentials of $30-60 \mathrm{mV}$ were obtained. In this case peripheral nerve bundle of the spinal ganglions was stimulated by an electric pulse. Sometimes the action potentials with the over-shoot were recorded (Fig. 2. B). However, they did not reach in many cases the zero potential level and the action potentials were of scores $\mathrm{mV}$. Those cases are thought that the cell body itself did not fire and the excitation of the membrane distant from the soma was electrotonically recorded at the cell body (1).

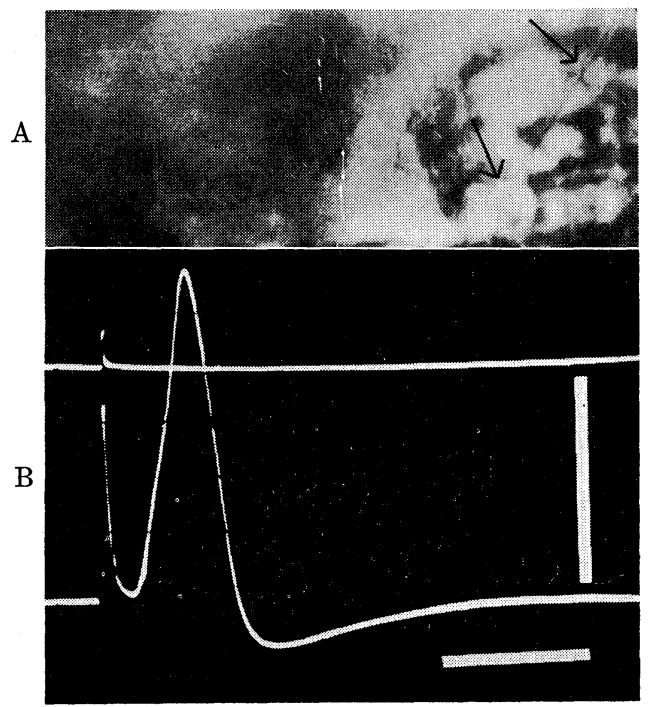

Fig. 2. Frog's spinal ganglion cell impaled by a microelectrode (A) and the action potential recorded from it (B). A pair of arrows in $\mathrm{A}$ show the microelectrode (upper) and the impaled cell (lower). The vertical and horizontal bars in B represent $50 \mathrm{mV}$ and 4 msec. respectively.

At the present stage it can be said that it is not difficult with this microscope to observe cells or nerve fibers which locate relatively superficially and it is possible to record their electrical activities with minimum injury due to the insertion of the prism and the illuminator into tissue. Further experiments are now under way on the nervous systems of higher animals, which have smaller cells.

During the process of the present study the other method with the same purpose was reported by Capellaro \& Kapany (2). However, 
they have not reported yet about the recording of the electrical activity of tissue cells.

The authors owe this work to Dr. Karl Frank of National Institutes of Health, U.S.A. and to Dr. Jun Koana, Professor of Optics, Department of Physics, of Tokyo University. We are very thankful for their continuous encouragement and precious advices. We are also indebted to Olympus Optical Co. and Sugiura Optical Research Laboratory for their elaboration on this work.

This work was supported by N.I.H. Research Grant B-2697.

\section{References}

[1] M. Ito: Jap. J. Physiol., 7, 297-323 (1957).

[2] D. F. Capellaro, and N.S. Kapany: Nature, Lond., 191, 927-928 (1961). 